\title{
An Efficient Method for Human Ear Recognition
}

\author{
Muhammad Sheikh Sadi ${ }^{*}$, Shaheena Sultana, Mostafizur Rahman and Md. \\ Rezwanul Islam \\ Department of Computer Science and Engineering, Khulna University of \\ Engineering and Technology, Khulna-9203, Bangladesh \\ E-mail: muhammad.sadi@graduate.curtin.edu.au
}

\begin{abstract}
Biometrics incorporates the investigation of programmed techniques for recognizing people in view of physical or behavioral qualities. Discovering great biometric techniques has been investigated widely in recent years. Among a few biometric techniques, ear is very steady since it doesn't differ with age and feeling. The ear is recognized based on the height of the ear, reference line cut point and corresponding angles. The proposed method is researched on the ear in random orientation and shows a greater accuracy than existing dominant approach. The recognition accuracy is increased by removing the noise in captured ear images and developing new methodologies to work with real time images.
\end{abstract}

Keywords: Ear recognition, Biometric security, Curve fitting, Ear orientation, Ear height line

\section{Introduction}

Driven by the requirements of various applications, for example, access control, flight security, or e- banking, naturally perceiving the personality of an individual with high certainty has turned into a subject of penetrating study. To take care of such an issue, biometrics identification, which utilize unique physical or behavioral attributes of individuals, are drawing expanding considerations now a days due to their high precision and robustness [1-2]. In this field, human identification is a great challenge in many commercial system and private system for authorized access [2]. Traditional identification system includes Personal Identification Number (PIN) and password, displaying ID, badges and so on. But traditional identification system has many disadvantages like PIN, password could be forgotten or hacked by masqueraders and ID badges could be lost [3]. This identification process can make the system vulnerable. Productive biometric techniques effectively manage those issues since clients are distinguished by their identity, not by something they need to recollect or convey with them. The latent techniques for biometrics don't require any activity from clients and can happen even without their insight. There are many known techniques for human identification based on image analysis. By and large, those biometric strategies can be partitioned into behavioral and physiological with respect to the source of data [4] and can be divided into passive and invasive biometrics, in regards to the way the information is procured [2]. The first class depends on the behavioral elements of human activities and it recognizes individuals by how they perform something. The most well-known of such strategies is voice verification. Different strategies are essentially in view of the elements of particular activities like signature, typing on the keyboard and simply moving or walking. Those strategies are not regular and they oblige clients to take part during the process of identification by rehashing particular activities, each time they are analyzed. Natural biometrics like face recognition, iris recognition, and finger print recognition has a great significance in this regard.

*Corresponding Author 
Ear fulfills the majority of the properties that ought to be controlled by a biometric, for example, uniqueness, all-inclusiveness, execution and collectability [4]. It has complex structure with uniqueness which is used to identify individuals. Even, ear of twins has different structure. In criminal investigation, ears are used as strong evidence [5]. In case of accidents, ears remain unchanged and could be used for investigation. Ear has been utilized for a long time in measurable sciences for acknowledgment [6]. Ear changes slightly in size and shape for persons' age [7]. It has not required any direct input to the authentication process.

Ear recognition has been done in several ways before. Almost all approaches are still facing the problem to reach an acceptable accuracy with real time images. There are huge gaps in the pre-processing stage of ear images as well. This paper proposes a methodology to recognize real time image of the ear with a better accuracy. Experimental studies validate the effectiveness of the proposed method. The proposed method outperforms the existing work in most of the real-life scenarios.

The paper is sorted out as follows; Section 2 discusses about related work on ear recognition, Section 3 contains the methodology of ear recognition system, Section 4 gives brief over view on experimental studies and concluding remarks are drawn in Section 5.

\section{Related Works}

Ear detection and identification can be done by several ways such as shape model based and ICP [8] and Helix Shape Model by Chen and Bhanu [9], connectivity graph by Prakash and Gupta [10], ICP using voxels by Yan and Bowyer [11], Cascaded AdaBoost by Abaza et al.. [12].

Chen and Bhanu [8] proposed three differing systems for ear recognizable proof. They arranged a classifier and perceived a specific distribution of shape records, which are the trademark for the ear's surface. In any case, this approach just takes a shot at profile pictures and sensitive to any kind of rotation, scale and posture variety. They likewise constrained the amount of possible ear candidates by distinguishing the skin area first before the helix template matching is connected on the curvature lines. In their augmented ear detection approaches, they recognized image region with an expansive local curvature and flow by a method which is called step edge magnitude [9]. At that point a layout, that contains the regular state of the external helix and the counter helix are fitted to bunch of lines. The principle constraint of Chen and Bhanu's proposed strategy [9] is the fixed data set. In arbitrary introduction, their strategy will neglect to identify the right individual.

A current approach on 2D ear acknowledgment using edges is portrayed by Prakash and Gupta in [10]. They combined skin division and grouping of edges into convex and concave edges. A while later, the edges in the skin area are deteriorated into edge fragments. These portions are made to frame an edge connectivity graph. In perspective of this graph, the convex hull of all edges, which are acknowledged to have a place with the ear, is prepared. The enclosed region is then named as the ear region. As opposed to [10], Prakash and Gupta demonstrated the practicality of edge-based ear recognition with respect to full profile pictures. They proposed a comparative edge availability for ear recognition on 3D pictures. As opposed to edges, they used discontinuities in the profundity outline separating the underlying edge picture and after that expelled the connectivity graph. In their examinations, they used the $3 \mathrm{D}$ representations of a vague subset as in [10]. Be that as it may, Discovery rate of their chart based approach is not in actuality by upset and scale.

Yan and Bowyer [11] developed an ear recognition procedure which wires range pictures and relating 2D color pictures. They started finding the concha and after that used dynamic forms for choosing the ear's external limit. The concha fills in as the reference point for putting the beginning state of the active contour model. Regardless of the way 
that the concha is definitely not hard to limit in profile pictures, it may be blocked on the off chance that the head posture changes or if a subject is wearing an amplifier or ear telephones. In their examinations, Yan and Bowyer [11] simply used ear pictures with minor impediments where the concha is obvious; consequently it couldn't be shown nor ruined whether their approach can do constantly recognizing ears if the concha is blocked.

Abaza et al. [12] and Islam et al. [13] used weak classifiers in light of Haar-wavelets with respect to AdaBoost [12] for ear localization. Abaza et al. [12] use an altered variant of AdaBoost and report a fundamentally shorter preparing stage. The feasibility of their approach is exhibited in evaluations on five distinct databases [12].For whatever length of time that the subject's stance does not change, weak classified ears are appropriate for pictures which contain more than one subject. They didn't say the real time recognition and their drawback was long period training phase. Heisele and Ritter [14] proposed a method for portioning transient groupings of range and power pictures. The mix of range and power information for division was grasped by clustering 4D force/position features. Kalman channels were then used to settle taking after by anticipating dynamic changes in group positions.

The ray transforms approach proposed by Alastair et al. [15]. It is expected to recognize the ear in different stances and to carelessness straight edges in the image, which can be displayed by glasses or hair. Ray transforms uses a light beam relationship to channel the picture for tubular and curved structures like the outer helix. The simulated beam is reflected in brilliant tubular regions and consequently these locales are highlighted in the changed image. Since glass outlines have straight edges, these are not highlighted by the ray change.

In 2005, Michał Choraś [16] suggested new methodology of ear recognition. Geometrical components that speaking to states of ear shapes are more reasonable for ear pictures than surface, color or global features. He regards the centroid as the particular point in this method, despite the fact that it is not a particular point inside the ear topology. Contour extraction may go wrong due to broken edge or inappropriate ear image. As a result, it will fail to determine the centroid of the contour image and break the continuity of the edges.

In 2012, Zahid et al. [2] al introduced new geometric method to recognize the ear. Their method was to find the ear edge using canny edge detection [17] from the static ear image and extract geometric features from the edge. They used three vectors as features, ear height line, reference lines and reference line cut point and corresponding angles. If the edge of the ear image is broken or vague then the whole methodology will fail. The ear orientation is another limitation of this method. If measured ear height line is inappropriate due to noisy edge and broken edge, the angle measured will also be incorrect.

\section{The Methodology of Ear Recognition System}

This paper proposes a 2D human-ear recognition framework to perceive the ear utilizing geometric elements in view of ear height line, reference lines and relating edges measured from outer ear edge. Preprocessing of ear image consists of several steps: Edge Detection, Noise Minimization by Curve Fitting and Area Calculation to Find the Ear Orientation. The stream outline of these means is showed up in Figure. 1 as proposed by Zahid et al. [2]. 


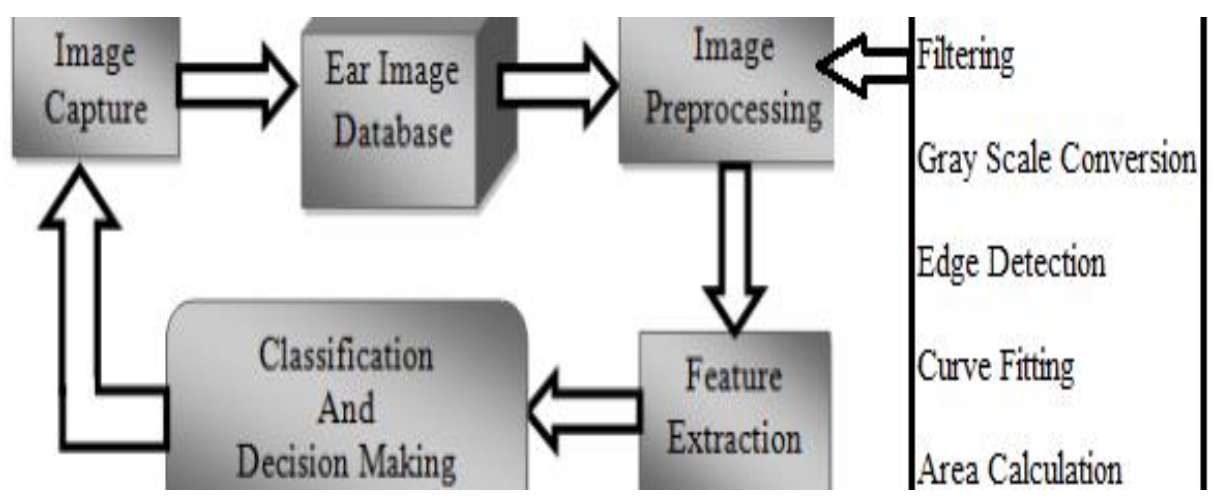

Figure 1. The Flow Diagram of Ear Recognition Methodology [1]

Reference lines are the lines which are parallel to the width of the photo and which fragment the photo cell into $(n+1)$ parts, where $n$ is a positive number. Figure 2 demonstrates the reference lines drawn in an ear image.

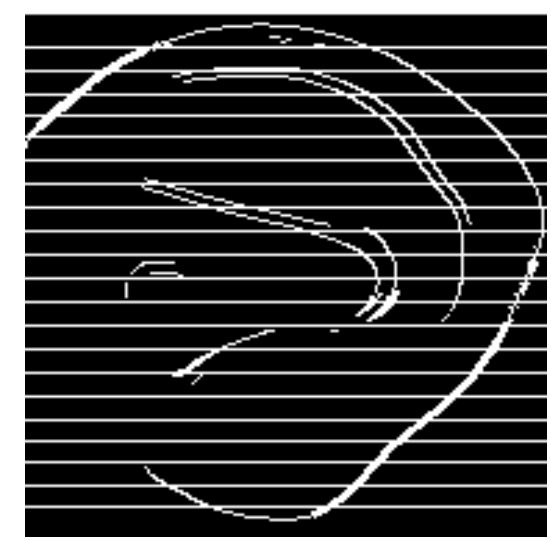

Figure 2. Reference Lines

The line that intersects bottom and top of the ear image is called ear height line. Figure 3 shows the ear height line of an ear image.

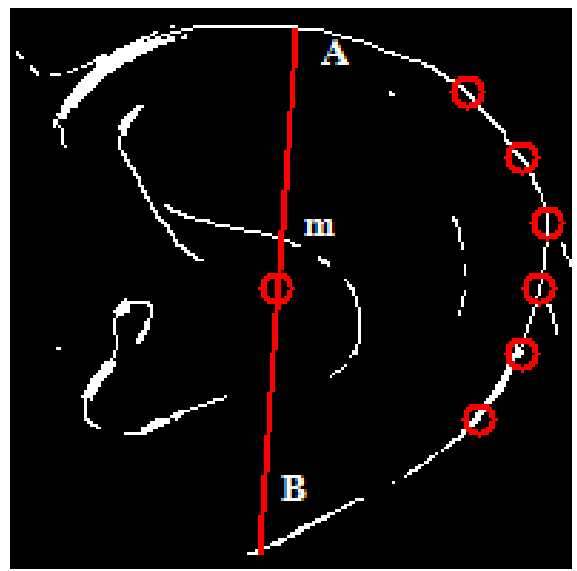

Figure 3. Ear Height Line

From the Figure 4, we see the co-ordinate of $b$ is $\left(b_{x}, b_{y}\right)$ which is the top point of ear edge and starting point of ear height line. Similarly co-ordinate of $a$ is $\left(a_{x}, a_{y}\right)$ which is the midpoint of ear height line. Here point $c\left(c_{x}, c_{y}\right)$ is the point produced by reference line 
cutting the outer ear edge. Hence, three points a, b, c is known but corresponding angle is unknown. Calculate ab, bc and ca using Euclidian distance by equation (1) is given by-

$$
a b=\sqrt{\left(a_{x}-b_{x}\right)^{2}+\left(a_{y}-b_{y}\right)^{2}}
$$

Then the cosine of the angle ${ }^{\theta}$ between $b a$ and $a c$ is taken from Figure 4 is given by equation (2).

$$
\cos \theta=\frac{\left\{(a b)^{2}+(a c)^{2}-(c a)^{2}\right\}}{2 * a b * a c}
$$

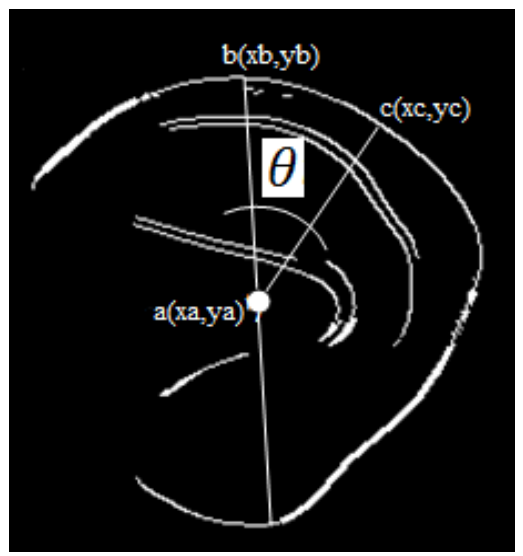

Figure 4. Angle Created by One Portion of Outer Ear Edge

Outer ear edge is very important to be detected clearly. If there is some noise, broken edge or incomplete outer edge then the whole methodology will fail. If the orientation of the image is changed, the methodology will not work properly. To overcome these limitations, ear images have to be processed more precisely. To fix broken edge or incomplete outer ear edge, 2nd degree curve fitting [17], namely parabolic curve fitting is introduced. To detect ear from random positions, it is needed to identify the proper orientation of the ear. To differentiate front ear image and rare ear image we need to calculate the area. In rear ear image, outer edge occupies a small area with the ear height line but in the case of front ear image it occupies greater area than the rear one. So by calculating the area between outer ear edge and ear height line the proper orientation of the ear image can be obtained.

\subsection{Noise Minimization by Curve Fitting}

Ear edges found from the image are not always perfect. So, the noise in the ear image should be removed. Edges are simple 2D curve and some portion may be broken.

In Figure 5, broken edges are indicated clearly. 


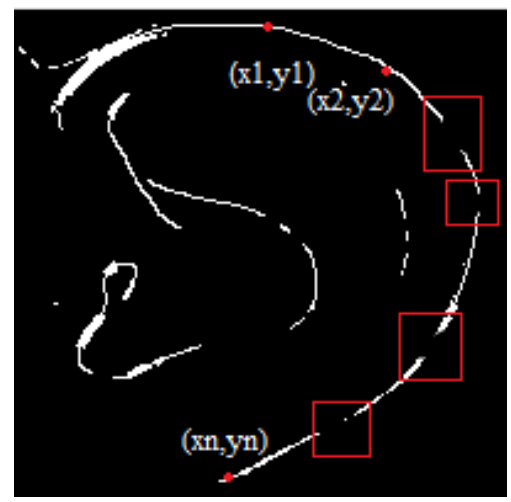

Figure 5. Ear Image with Broken Edge

The positions of each pixel are found in 2D co-ordinate system. Then binomial equations are solved to find the value of the co-efficient and fit the curve properly. The outer edge of the ear is in parabolic shape. Now, the equation may be found from the outer ear edge as follows:

$$
y=a+b x+c x^{2}
$$

Here, $a, b$ and $c$ are three co-efficient in which outer ear edge equation depends on. Equation (3) satisfies given data

$$
\left(x_{1}, y_{1}\right),\left(x_{2}, y_{2}\right),\left(x_{3}, y_{3}\right) \ldots \ldots \ldots\left(x_{n}, y_{n}\right)
$$

That is the visible outer ear edge points.

Let, $y_{\lambda}$ be the theoretical value for $x_{1 \text { then, }}$

Error,

$$
\begin{aligned}
& e_{1=} y_{1}-y_{\lambda} \\
\Rightarrow & e_{1}=y_{1}-\left(a+b x_{1}+c x_{1}^{2}\right)^{1} \\
\Rightarrow & e^{2}{ }_{1}=\left(y_{1}-a-b x_{1}-c x^{2}{ }_{1}\right)^{2}
\end{aligned}
$$

Let,

$$
\begin{gathered}
\operatorname{Sum}_{\mathrm{n}} S=\sum_{\mathrm{i}=1}^{\mathrm{n}} e_{i}^{2} \\
\sum_{\mathrm{i}=1}^{2}\left(y_{i}-a-b x_{i}-c x_{i}^{2}\right)^{2}
\end{gathered}
$$

By the principle of least square [17], minimum value of $S$ is 0 . Now, getting partial derivative of $S$ with respect to $a, b$ and $c$, we get-

$$
\frac{\delta S}{\delta a}=0, \quad \frac{\delta S}{\delta b}=0, \quad \frac{\delta S}{\delta c}=0
$$

Solving the equation (2) and dropping the suffix we get

$$
\begin{aligned}
& \sum y=\mathrm{n}^{a}+b^{\sum x}+c^{\sum x^{2}} \\
& \sum x y=a \sum x+b^{\sum x^{2}}+c^{\sum x^{3}} \\
& \sum x^{2} y=a \sum x^{2}+b^{\sum x^{3}}+c^{\sum x^{4}}
\end{aligned}
$$

The equation (5), (6) and (7) are known as normal equation and solving the equation (5), (6) and (7) we get the value of $a, b$ and $c$. Putting the value of $a, b$ and $c$ in the equation (1), outer ear edge can be obtained and then curve fitting can be introduced in the broken edge curve. It is possible to extract geometric feature from the image of the outer ear at any orientation. 


\subsection{Ear Orientation and Random Positioning}

Two ear images shown in Figure 6 are examined, one is front ear image and another one is back ear. Front ear image occupies larger geometric area than the rear one. Comparing their area with a fixed threshold value front and rear ear can be distinguished easily. The threshold value is taken empirically. Figure 6 also shows the ear height line and reference line cut point for both rear and front end.

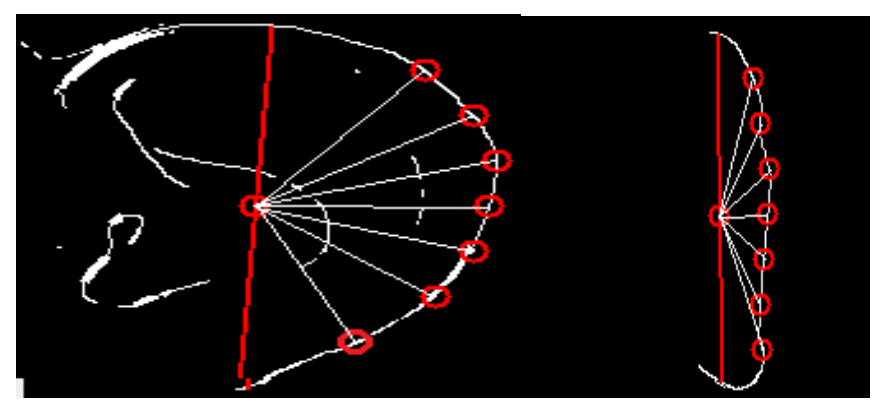

Figure 6. Front Ear and Rear Ear Image

Let, the equation of the ear height line be

$$
y=b+m x
$$

Where, $\mathrm{m}$ is slope of the height line.

Let the equation of the outer ear edge curve be

$$
y=a+d x+c x^{2}
$$

Solving (8) and (9), the two values of $\mathrm{x}$ are found. After the limit is being found, the equation (9) is integrated between the limit of $x$ and the outcome is the area between ear height line and outer edge of the ear.

So, area is calculated by the following equation

$$
A=\int_{\min o f x}^{\max o f x} y d x
$$

Where,

$$
y=a+d x+c x^{2}
$$

Value of $y$ is calculated as equation (3). Observing the value of the calculated area, $A$; orientation of the ear is found by comparing it with threshold value. Threshold value is taken empirically.

\subsection{Ear Recognition System}

Arrangement is performed in three phases.

In first phase, the first main component vector is utilized. Ear height line is utilized as the first component vector.

Let, $V 1_{1}=\left[\Delta_{1}\right]$ and $V 1_{2}=\left[\partial_{1}\right]$ be the first component vectors of two pictures. Using this vector $D_{1}$ is calculated.

$$
D_{1}=\left|\partial_{1}-\Delta_{1}\right|
$$

Here, $w_{1}$ is the number of data that match. Two data are said to be matched if their corresponding distances are same. Number of matched data,

$$
w_{1}=\sum_{i=1}^{n} x_{i}
$$

Where $\mathrm{x}$ is an integer which can have just two values.

if $\left|\partial_{1}-\Delta_{1}\right|$ is less than threshold value then 


$$
\begin{gathered}
x_{i}=1 \\
\text { else } \\
\mathrm{x}_{\mathrm{i}}=0
\end{gathered}
$$

In second phase, second component vectors are utilized. Second component vector comprises of the estimation of the distances of $n$ points from the center of the ear height line comparing to $\mathrm{n}$ points of the outer edge.

$$
\text { Let, } V 2_{1}=\left[\alpha_{1}, \alpha_{2}, \alpha_{2}, \ldots \ldots, \alpha_{n}\right] \text { and } V 2_{2}=\left[\beta_{1}, \beta_{2}, \beta_{3}, \ldots \ldots, \beta_{n}\right]
$$

Be the second component vectors of two images. Using the difference between $V 2_{1}$ and $V 2_{2}$ the second component vector $D_{2}$ is calculated. If the distances are same [in the case of identical images] then $D_{2}$ equals to 0 .

$$
D_{2}=\sum_{i=1}^{n}\left|\alpha_{i}-\beta_{i}\right|
$$

Here, ${ }^{w_{2}}$ is the number of distances where $D_{2}$ is 0 or less than threshold value.

$$
w_{2}=\sum_{i=1}^{n} y_{i}
$$

if $\left|\alpha_{i}-\beta_{i}\right|$ is less than threshold value then

$$
\begin{gathered}
y_{i}=1 \\
\text { else } \\
y_{i}=0
\end{gathered}
$$

Two pictures are said to be matched as for the primary component vector if $D_{2}$ and $w_{2}$ are less than some threshold values.

In the third phase, third component vector is utilized. In this stage two points are said to be matched if their corresponding angles are around same and they relate to a similar reference line.

Let $w_{a}$ denote the number of points that are matched i.e. two corresponding angles are matched then $w_{a}$ is incremented by 1 . But as the size of second component vector is not fixed the percentage of matched points $D_{a}$ is calculated by the equation (14).

$$
D_{3}=\frac{w_{3}}{\min \left(v_{1}, v_{2}\right)}
$$

Here $v_{1}, v_{2}$ are sizes of third component vectors of two images.

Two pictures are said to be matched at last on the off chance that they are matched concerning first component vector, second component vector and $\mathrm{D}_{3}$ is not as much as threshold value. In this way a given request picture is at first tried against each one of the pictures in the database using first component vector. Simply the pictures that are matched in the primary phase are considered for the second phase of arrangement and the pictures that are matched in the second phased are considered for the third phase of grouping. Along these lines division of characterization into three phases fundamentally decreases the time taken for arrangement.

\section{Experimental Analysis}

The method is implemented in Microsoft Visual Studio 2012. Canny Edge detection algorithm [16] is used here for finding the ear edge. For real time identification ear images are captured by Microsoft webcam and particular driver software is used for this purpose. Approximately sixty ear images are taken for experiment. Real time ear images are processed according to methodology and then raw images with edges are found as output of the system and curve fitting are applied to the system. Three feature vectors that are used in this methodology are ear height line, reference lines and the corresponding 
angles. Finally, all three feature vectors are extracted and compared with the database of saved ear images and traced out the exact match. Ear orientation and edge correction are shown in Figure 7.

In this paper the extracted features are all distances and corresponding angles. These features are divided into three vectors. The first feature vector is the height of the ear, second feature vector is the distances measured from the reference lines' cut points and midpoint of the ear height line and third feature vector is the collection of corresponding angles. Angles depend on the outer edge of the ear image and other two types of distances are found from reference lines and ear height line. Area is calculated by integrating the outer ear curve and ear height line.

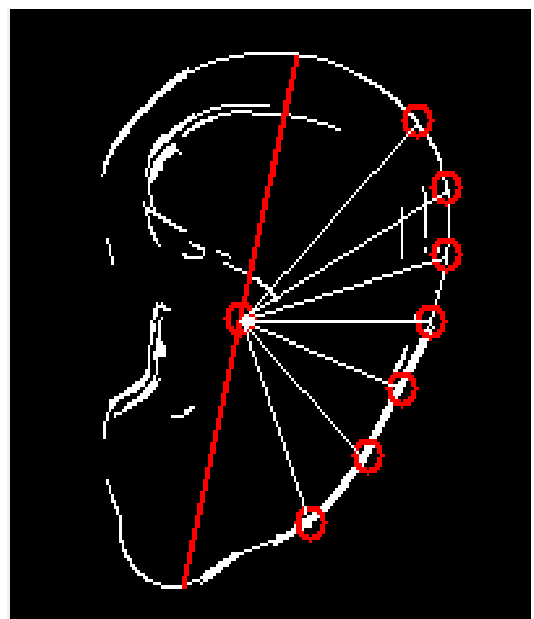

Figure 7. Experimental Ear Image

Table 1 contains area, fitted points and ear height line for the ear image as shown in Figure 7.

Table 1. Area, Fitted Points and Ear Height Line

\begin{tabular}{|c|c|c|}
\hline $\begin{array}{c}\text { Area } \\
\text { [front] }\end{array}$ & $\begin{array}{c}\text { New points } \\
\text { [fitted points] }\end{array}$ & Ear Height line \\
\hline 3.353 & $(90,178)$ to 101,174$)$ & 180.44666802133 \\
\hline
\end{tabular}

Table 2 contains 2nd component vector. [D1, D2 ... D7] are the 2nd component vector that indicates the distances between reference line cut point and the midpoint of the ear height line. These values are compared to decide which image is matched most. During comparing with the saved database, threshold value is also considered for acceptance and rejection. It helps to minimize the total comparisons as well. Values of 2 nd component vector from Figure 7 are extracted and shown in the Table 2. 


\section{Table 2. Distances Between Reference Lines' Cut Points and Ear Height} Line Mid-Point

\begin{tabular}{|c|c|}
\hline Distances & Value \\
\hline D1 & 89.7385090136893 \\
\hline D2 & 94.0691235209513 \\
\hline D3 & 89.9444272870754 \\
\hline D4 & 73.0068489937759 \\
\hline D5 & 66.1286624694618 \\
\hline D6 & 66.5281895139196 \\
\hline D7 & 72.2357252334328 \\
\hline
\end{tabular}

Table 3 contains 3 rd component vector. 3rd component vectors are angles [A1, A2, A3 ... An] produced in the ear height line midpoint by the reference lines' cut points. Angles as 3rd component vector are shown in Table 3.

A given inquiry picture is at first tried against each one of the pictures in the database using first component vector. Simply the pictures matched in the first phase are considered for the second phase and the pictures matched in second phase are considered for third phase of characterization. As the measure of first component vector size is 1, since for this circumstance the proposed procedure will perform one correlation. The amount of reference lines are $n$, so the amount of correlations required for the second phase of grouping are $n$. In the third phase of characterization, $m^{*} n$ relationships are required, expecting $m$ comparing plots for $n$ reference lines.

Let ${ }^{I}$ be the number of images in the database. If the classification is single stage then required comparisons are $[I *\{1+(n)+(m * n)\}]$. For each of $I$ images, one comparison for first feature vector, ${ }^{n}$ for second and ${ }^{m * n}$ comparisons for third feature vector.

Table 3. Corresponding Angles by Reference Lines Cut Points and Ear Height Line

\begin{tabular}{|c|c|}
\hline Corresponding Angle & Value \\
\hline A1 & 32.101070337226 \\
\hline A2 & 15.1482126354302 \\
\hline A3 & 13.6733536293023 \\
\hline A4 & 15.6710914520095 \\
\hline A5 & 19.5684045450769 \\
\hline A6 & 22.2101226051210 \\
\hline A7 & 25.4879491632832 \\
\hline A8 & 35.9849425514534 \\
\hline
\end{tabular}


Because of the three phases grouping the effective numbers of examinations are reduced $\left.^{\left[I+\left(I_{1} * n\right)\right.}+\left\{I_{2} *(m * n)\right\}\right]$ is the quantity of pictures that are matched as for first component vector and $I_{-2}$ is the quantity of pictures that are matched regarding second

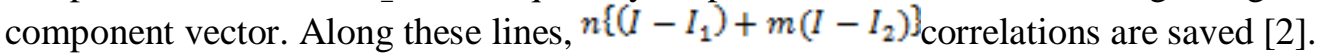

Zahid et al. [2] worked with static images which are captured from front view. Ear is recognized in fixed position. They don't trace out ear images from real environmental images. It was inappropriate for random ear orientations, real time detection and recognition. Without introducing curve fitting and changing the ear orientation, Zahid et al. [2] reduces its accuracy at a huge rate. Applying random positioning of the ear image and without curve fitting it becomes $91.37 \%$ accurate. Applying advance image processing, this proposed method has increased its accuracy to $96.8 \%$. Figure 8 illustrates comparison between Zahid et al. [2] and proposed method.

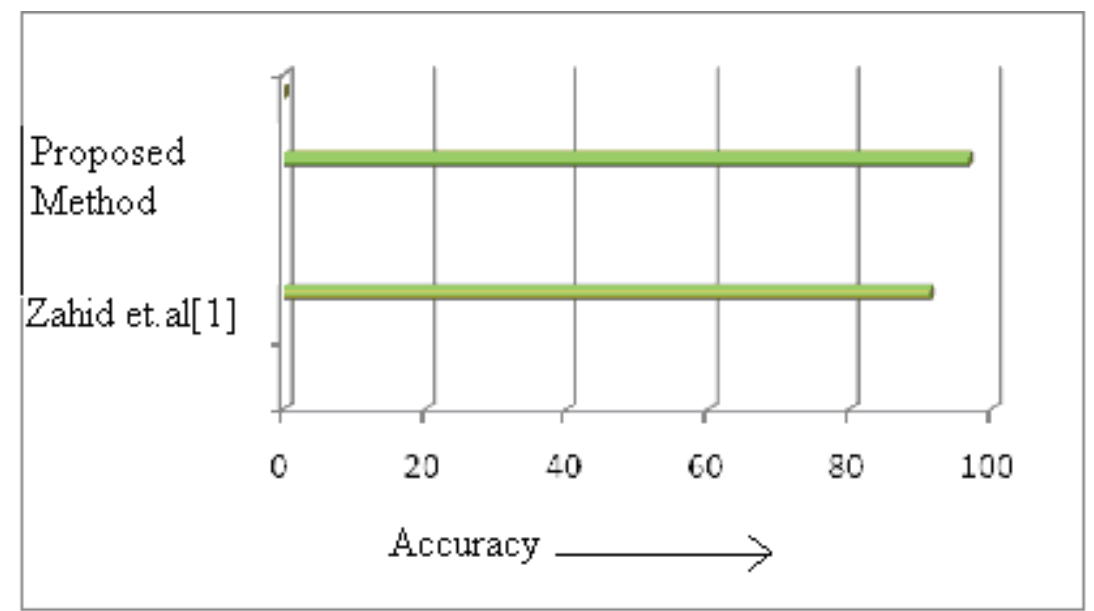

\section{Figure 8. Comparison between the Proposed Method and the Method Shown By Zahid et al. [2]}

Choosing the incentive for number of reference lines $\mathrm{n}$ is a key perspective in this approach. A more noticeable estimation of $\mathrm{n}$ grows the exactness also builds the space prerequisite and the time taken for order as more number of elements is to be analyzed. In this way an ideal esteem ought to be picked which satisfies all the three requirements of space, time and precision. Error rate comparison with Zahid et al. [2] is shown in Figure 9.

Choosing the motivating force for number of reference lines $\mathrm{n}$ is a vital point of view in this approach. A more unmistakable estimation of $n$ broadens the exactness in addition manufactures the space essential and the time taken for characterization as more number of components is to be analyzed. Along these lines a perfect ideal incentive to be picked which fulfills all the three basics of space, time and precision. 


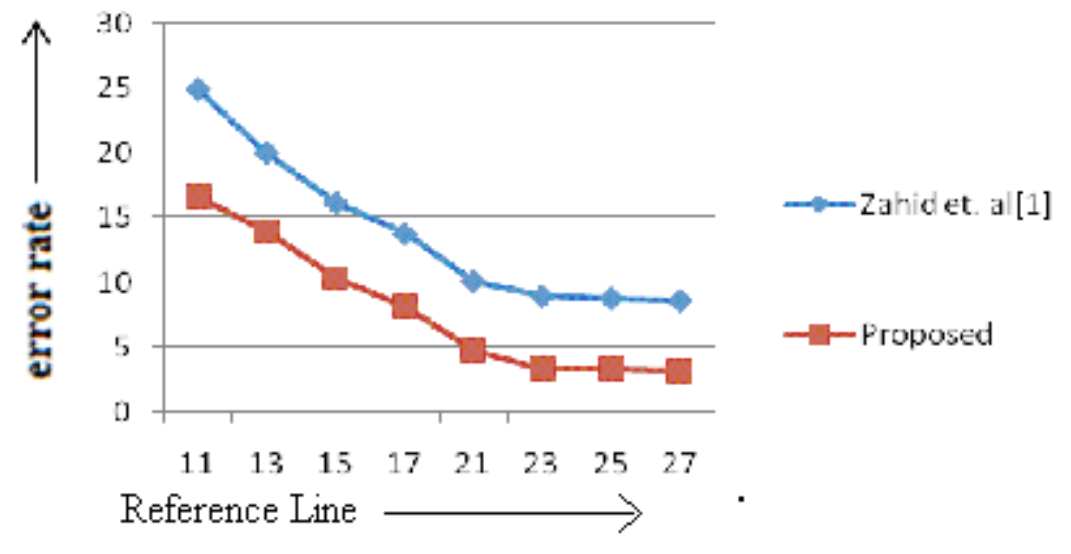

Figure 9. Graphical Representation of Our Experimental Result

Figure 9.shows that after taking 21, 23 and 27 reference lines the error rate reduces linearly. But the main fact is that when curve fitting is introduced in this proposed method, accuracy increased and when random positioning is introduced in earlier methods, its accuracy falls drastically which was overcome in this proposed methodology. Proposed methodology proves its best result experimentally.

\section{Conclusions}

This paper proposes a new methodology to perceive ear with a real time random orientation. Moving person's ear can be captured and processed through this proposed method and can be traced out with a better accuracy than existing domain approach. This process includes curve fitting of the outer ear edge and finding the proper orientation of the ear. Although proposed methodology works weakly in low hysteresis threshold, it recognizes the ear more accurately and accustomed with the real environment. Moving ear image is recognized and classified through this method and it can identify individual more accurately. There is some complexity capturing real time images, because captured image may not always contain the ear. This work can be extended for inner curve fitting of the ear and extracting features of the inner portion of the ear edge.

\section{References}

[1] Z. Lin , L. Lida, L. Hongyu, Y. Meng, "3D Ear Identification Using Block-Wise Statistics-Based Features and LC-KSVD," IEEE Transactions on Multimedia, Volume: 18, Issue: 8, Page: 1531 - 1541, May 2016.

[2] Md. Zahid Hasan Polin, A. N. M EnamulKabir, Muhammand Sheikh Sadi, "2D Human-ear Recognition Using Geometric Features," in Proc. IEEE Int. Conf. on Electrical and Computer Engineering, Dec. 2012.

[3] M. Choras, "Ear biometrics based on geometrical feature extraction", Electronic Letters on Computer Vision and Image Analysis, May, 2005, pp. 84-95.

[4] Choras M., "Perspective Methods of Human Identification: Ear Biometrics," Opto-Electronics Review. 2008, pp. 16:85-96.

[5] A. Iannarelli, "Ear Identification, Forensic Identification Series", Paramount Publishing Company, 1989

[6] Chen H, Bhanu B, "Shape Model-Based 3D Ear Detection from Side Face Range Images," in Proc. IEEE Int. Conf. Computer Vision and Pattern Recognition., Jun. 2005, pp. 122.

[7] Meijerman L, Van Der Lugt C, Maat GJR, “Cross-sectional anthropometric study of the external ear," J Forensic Science, 286-293,2007.

[8] Chen H, Bhanu B. "Human Ear Recognition in 3D". IEEE Transactions on Pattern Analysis and Machine Intelligence. 2007 April; 29(4):718-737.

[9] Chen H, Bhanu B., "Contour Matching for 3D Ear Recognition," in Proc. Of seventh IEEE Workshop on Application of Computer Vision (WACV/MOTION);Jun. 2005. 
[10] Prakash S, Gupta P. An Efficient Ear Localization Technique. Image and Vision Computing. 2012; 30:38-50.

[11] Yan P, Bowyer KW. "Biometric Recognition Using 3D Ear Shape". Pattern Analysis and Machine Intelligence. 2007 August; 29 pp.1297-1308.

[12] Abaza A, Hebert C, Harrison MAF, "Fast Learning e Ear Detection for Real-time surveillance," in Proc. IEEE Int. Conf. Biometrics: Theory Applications and Systems(BTAS-2012) ., Apr.2010, pp. 1-6.

[13] Islam S, Davies R, Bennamoun M, MianA.'"Efficient Detection and Recognition of 3D Ears". International Journal of Computer Vision. 2011;95:52-73.

[14] Ping Yan and Kevin W. Bowyer, "Ear biometrics using 2D and 3D images”, CVPR '05, page 121, Washington, DC, USA, 2005, IEEE Computer Security.

[15] Alastair H, Cummings AH, Nixon MS, Carter JN. "A Novel Ray Analogy for Enrol-ment of Ear Biometrics". In: Fourth IEEE International Conference on Biometrics: Theory Applications and Systems (BTAS); 2010.

[16] Michał Choraś, "Contour Matching for 3D Ear Recognition,” in Proc. IEEE Int. Conf. Electronic Letters on Computer Vision and Image Analysis., Jan. 2005, pp. 87.

[17] J. Canny, "A Computational Approach to Edge Detection," IEEE Trans. on Pattern Analysis and Machine Intelligence, 8(6), pp. 679-698, 1986.

[18] Curve Fitting and Solution of Equation. [Online]. Available at:http://www.newagepublishers.com/samplechapter/001938.pdf. 
International Journal of Signal Processing, Image Processing and Pattern Recognition Vol. 10, No. 4 (2017) 